\title{
Modelo de gestão em incubadoras e mapas de desempenho
}

\section{Management model in incubators and performance of maps}

\author{
Lieda Amaral Souza ${ }^{1}$ \\ Pablo Fernandes Dantas ${ }^{2}$ \\ Walid Abbas El Aouar ${ }^{3}$ \\ Lais Karla Silva Barreto ${ }^{4}$
}

\section{Resumo}

O estudo objetiva discutir a proposta de um modelo de gestão aplicável às incubadoras, tendo por base os mapas estratégicos e painéis de desempenho à luz das premissas do Balanced Scoredcard (BSC) e utilizá-lo como ferramenta de medição de desempenho das empresas incubadas de acordo com as práticas-chave do modelo CERNE. Adotou-se abordagem qualitativa desenvolvendo-se um estudo de caso em uma incubadora de base tecnológica, utilizando-se da técnica Delphi para construção dos objetivos estratégicos, os quais foram concebidos para composição do Mapa e do Painel de Indicadores de Desempenho. Os achados da pesquisa apresentam uma proposta inicial de mapa e painel de desempenho contemplando as quatro perspectivas propostas pelo BSC.

Palavras-Chave: Incubadora. BSC. Mapas estratégicos. CERNE.

\section{Abstract}

This paper aims to discuss the proposal for a management model applicable to incubators based on the strategic maps and performance dashboards according

Pesquisadora. Mestre em Engenharia da Produção e Doutoranda em Tecnologia e Sistemas de Informações. EEng/PDTSI/Uminho. Campus de Azurem - Guimarães - Portugal. lieda@dsi. uminho.pt

2 Pós-Graduado em Controladoria e Finanças pela UFRN. pablo.fernandes@gmail.com

3 Professor e Coordenador do Mestrado Profissional em Administração da UnP, Mestre e Doutor em Administração, Pesquisador do Comportamento Organizacional. walidabbas@unp.br

4 Doutora em Estudos da Linguagem pela Universidade Federal do Rio Grande do Norte (UFRN), docente do Mestrado Profissional em Administração da Universidade Potiguar. Endereço: Av. Eng. Roberto Freire, 1684, Capim Macio, CEP: 59.082-902, Natal-RN; Fone: (84) 3216-8616. laisbarreto@gmail.com 
the premises of the Balanced Scorecard (BSC) and use it as a performance measurement tool of the incubated companies according to the key practices CERNE model. It was adopted a qualitative approach by developing a case study in a technological incubator, using the Delphi technique for construction of the strategic objectives which are designed to map the composition and Performance Indicators Panel. The research findings present an initial proposal for map and performance dashboard contemplating the four perspectives proposed by the BSC (Learning and Development, Internal Processes, Customers and Financial). It is therefore an innovative approach to relationship management and performance evaluation between the incubator and companies affected by the scheme.

Keywords: Incubator. BSC. Strategy maps. CERNE.

\section{Introdução}

Nas últimas décadas, o Brasil registrou, no cenário econômico e social, avanços representativos e significativos. Essa nova configuração econômica, em grande parte, é resultado da contribuição das micro e pequenas empresas (MPE), responsáveis pela geração de novos empregos e aumento da renda, possibilitando a melhoria da qualidade de vida. Nos últimos resultados divulgados pelo IBGE (2013), as MPE representam $60 \%$ dos 94 milhões de empregos do país e são responsáveis por $20 \%$ do PIB nacional. No entanto, mesmo diante dessa realidade favorável, existe uma série de fatores que são causadores do insucesso dessas empresas como a ausência de planejamento, a falta de conhecimento de mercado de atuação e de organização empresarial.

O papel das incubadoras de empresas é estimular a criação e o desenvolvimento de novas ideias de negócio, fornecendo uma estrutura de apoio e assessorias para empresas nascentes, oferecendoIhes serviços em consultoria especializada, capacitação gerencial e orientação necessária para elaboração dos projetos, com o objetivo de ampliar as chances de sucesso empresarial.

Diante desse cenário, um dos principais desafios das incubadoras de empresas é alinhar as atividades da organização às suas estratégias. Desse modo, surge a necessidade de uma ferramenta operacional 
que ofereça mais do que indicadores de desempenho tradicionais e que permita delinear um direcionamento estratégico. O Balanced Scorecard (BSC) é um modelo de gestão estratégica desenvolvido pelos americanos Kaplan e Norton, nos anos de 1990, apresentando uma nova filosofia de gestão em que as perspectivas financeiras são equilibradas por perspectivas não financeiras; com isso, rompendo paradigmas dos tradicionais métodos. O BSC se propõe a traduzir a visão e a estratégia da organização em objetivos operacionais, direcionando o seu desempenho e tornando possível gerenciar a estratégia de maneira integrada, garantindo que os esforços estejam todos voltados ao foco estratégico.

Atualmente, as incubadoras do Brasil priorizam a implantação do Modelo de Gestão de Incubadoras (CERNE), buscando a certificação por meio da implementação de suas práticas. A implantação das práticas-chave do CERNE é um processo que envolve todas as áreas de atuação das incubadoras, proporcionando o monitoramento e avaliação do desempenho, através de uma série de indicadores. Nesse contexto, o objetivo desta pesquisa é discutir a proposta de um modelo de gestão aplicável às incubadoras, tendo por base os mapas estratégicos e painéis de desempenho à luz das premissas do Balanced Scoredcard (BSC) e utilizá-los como ferramenta de medição de desempenho das empresas incubadas de acordo com as práticas-chave do modelo CERNE.

Adotou-se para desenvolvimento do estudo uma abordagem qualitativa desenvolvendo-se um estudo de caso em uma incubadora de base tecnológica, utilizando-se da técnica Delphi para construção dos objetivos estratégicos, os quais foram concebidos para composição do Mapa e do Painel de Indicadores de Desempenho, por possibilitar um processo participativo, em que os referidos objetivos estratégicos escolhidos para composição do mapa foram repetidos a cada rodada, de modo que os participantes da incubadora pudessem reavaliar as suas respostas e as justificar. Embora existam pesquisas sobre avaliação de desempenho em algumas incubadoras, o estudo se justifica posto que não existem evidências empíricas sobre quais as melhores técnicas de mensuração de desempenho adaptáveis ao contexto das incubadoras. 
Os resultados da pesquisa apresentam uma proposta inicial de mapa e painel de desempenho contemplando as quatro perspectivas propostas pelo BSC (Aprendizagem e Desenvolvimento, Processos Internos, Clientes e Financeira). Trata-se, portanto, de uma abordagem inovadora da gestão do relacionamento e de avaliação de desempenho entre a incubadora e as empresas beneficiadas pelo sistema.

\section{O Modelo CERNE}

O Manual de Implantação do Modelo de Gestão de Incubadoras (CERNE) surgiu por meio da parceria entre a Associação Nacional das Entidades Promotoras de Empreendimentos Inovadores (ANPROTEC) e o Serviço Brasileiro de Apoio às Micro e Pequenas Empresas (SEBRAE). O projeto tem o objetivo de expandir, por meio de uma plataforma de auxílio e soluções, o número de empreendimentos de sucesso desenvolvidos em incubadoras que passam a contar com uma estrutura de referência em gestão, minimizando os riscos de fracasso na formação de novos negócios e o processo de incubação de empresas torna-se sistematizado (ANPROTEC, 2011).

A aplicação desta metodologia de gestão nas incubadoras busca prioritariamente a implantação de suas práticas-chave. A implementação das práticas permite às incubadoras atrair e selecionar novos empreendimentos e monitorá-los para o seu rápido desenvolvimento, por meio de indicadores de desempenho elaborados com o processochave 1.6, Sistema de Acompanhamento, Orientação e Avaliação do modelo CERNE.

A avaliação e o monitoramento das empresas estão relacionados às cinco práticas-chave apresentadas pelo manual do CERNE: empreendedor, tecnologia, mercado, finanças e gestão.

Segundo o Manual de Implantação do CERNE (2011), as práticaschave referentes ao processo-chave de número 1.6 definem-se como:

- Monitoramento do Empreendedor: permite acompanhar o desenvolvimento do empreendedor; 
- Monitoramento da Tecnologia: permite acompanhar o desenvolvimento do produto, serviço e tecnologia.

- Monitoramento do Mercado: permite acompanhar o desenvolvimento comercial dos empreendimentos;

- Monitoramento Financeiro: permite acompanhar os processos de geração e captação de recursos financeiros dos empreendedores;

- Monitoramento da Gestão: permite acompanhar o desenvolvimento da gestão dos empreendimentos.

O projeto possibilita melhorias na gestão das incubadoras, além de garantir sua sustentabilidade e crescimento, atraindo visibilidade e integração com seus parceiros e comunidade.

\section{BSC: Mapas Estratégicos}

O Balanced Scorecard (BSC) surgiu em princípios da década de 1990, por meio de trabalhos publicados pelos americanos Robert Kaplan e David Norton. Os modelos de gestão, até então existentes, contemplavam apenas indicadores financeiros, incapazes de refletir os resultados de projeções de desempenho futuros. O BSC trouxe uma nova filosofia de gestão que faz uso do equilíbrio entre os indicadores financeiros e não financeiros diferenciando-se dos tradicionais métodos utilizados. Segundo Kaplan e Norton (1997), o Balanced Scorecard é uma ferramenta que mede o desempenho organizacional sob quatro perspectivas distintas e equilibradas, no entanto interligadas: Aprendizagem e Desenvolvimento, Processos Internos, Clientes e Financeira. De tal maneira, o BSC oferece um acompanhamento do desempenho financeiro, ao mesmo tempo, que monitora a formulação da capacidade e a aquisição dos ativos intangíveis, fundamentais para o seu desenvolvimento em longo prazo.

BSC é uma sigla, que traduzida, significa Indicadores Balanceados de Desempenho e avalia que a escolhas dos indicadores para a gestão de uma organização não se restringem apenas aos dados econômicofinanceiros. Também se faz necessário que as organizações monitorem 
indicadores com foco não financeiros tais como: desempenho de mercado junto a clientes, desempenho dos processos internos e pessoas, tecnologia e inovação. A somatória destes indicadores alavancará o desempenho esperado e criará valor futuro.

A percepção do Balanced Scorecard permite que as organizações tornem-se orientadas à estratégia. Para isso, cinco princípios auxiliam para que o alinhamento e o foco estratégico estejam conservados, ainda que as realidades das organizações sejam distintas:

- Traduzir a estratégia em termos operacionais: permite que todos os integrantes da organização possam entendê-la;

- Alinhar a organização à estratégia: permite o envolvimento e comprometimento de toda organização;

- Transformar a estratégia em tarefa de todos: permite por meio da contribuição pessoal a implementação da estratégia;

- Converter a estratégia em processo contínuo: permite o avanço do processo estratégico através do aprendizado e revisões contínuas da estratégia;

- Mobilizar a mudança por meio da liderança executiva: permite promover a transformação estratégica.

A Figura 1 apresenta os princípios da organização orientada para a estratégia, com enfoque nos preceitos do BSC.

Figura 1 - Princípio da Organização Orientada para a Estratégia

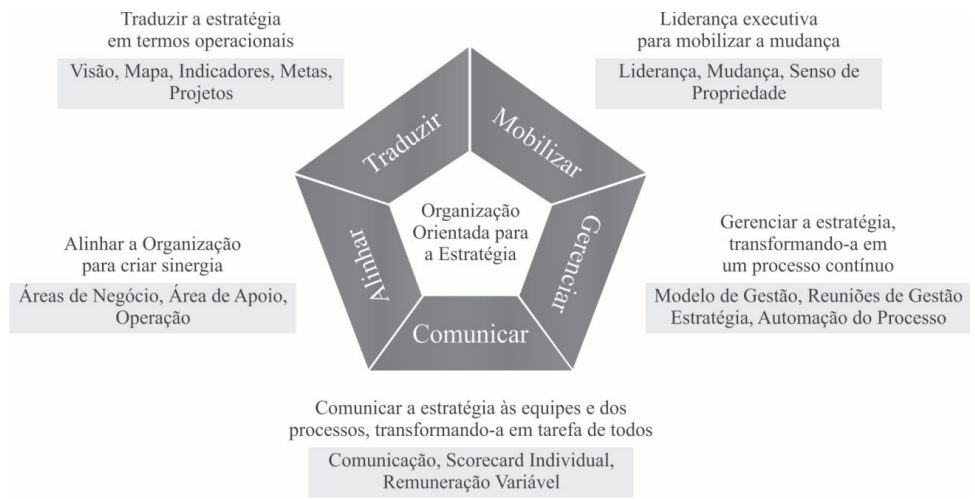

Fonte: Adaptado de Kaplan e Norton (2000) 
O BSC apresenta a visão e a estratégia traduzidas em objetivos operacionais. Como um processo vivo de contínuo aprendizado, esta ferramenta vem crescendo no decorrer dos anos, tornando-se uma referência em termos de mensuração de desempenho para a gestão estratégica das organizações.

Os mapas estratégicos relatam as relações de causa e efeito pertencentes ao alcance da visão institucional e monitoram suas atividades gerais com base em uma única direção estratégica (REZENDE, 2003). No entanto, os mapas estratégicos são uma ferramenta de representação visual, que permite descrever a estratégia da organização de maneira única e consistente, coordenando-a para atingir os seus objetivos e conectá-los aos resultados. Tal concepção é admitida por Kaplan e Norton (2004), ao relatarem que os mapas estratégicos formam os pilares para a construção do Balanced Scorecard e estão conectados à estratégia da organização (GUIMARÃES; SILVA FILHO, 2007).

$\mathrm{Na}$ visão de seus autores, a partir de sua estruturação os mapas estratégicos podem explicitar o processo desta transformação intangível em resultados tangíveis, uma vez que aponta como a organização projeta transformar seus ativos em resultados almejados, além de conceder uma referência para descrever e gerenciar sua estratégia. Dessa forma, os mapas auxiliam as organizações a delinearem suas estratégias de modo integrado e sistemático, oferecendo um alicerce ao sistema gerencial.

Além do desdobramento nas perspectivas ilustradas (aprendizagem e desenvolvimento, processos internos, clientes e financeira), faz-se necessário o uso de informações que efetivamente auxiliem o processo de alinhamento estratégico. Isso se faz por meio da construção de um painel de desempenho, em que constam as perspectivas, os objetivos estratégicos, os indicadores de desempenho, as metas e as iniciativas sugeridas para o alcance das metas (IGARASHI et al., 2010). Após a construção do painel de desempenho, o BSC passa a possui caráter gerencial, devido à contribuição no processo de gerenciamento.

Conh e Khurana (2003), Costa (2006) e Fernandes e Berton (2005) consideram o processo de gerenciamento mais amplo do 
que apenas acompanhar se as metas serão atingidas, ou não, mas também auxiliar na interligação e integração das estratégias individuais, tornando o todo mais representativo do que as somas das partes. Nesse âmbito, Kaplan e Norton (2006) admitem a sinergia como um diferencial na operacionalização do mapa estratégico, ao proporcionar aos participantes a percepção da visão estratégica, possibilitando que eles atuem de maneira participativa em relação às metas propostas.

\section{Integração do BSC versus CERNE}

O Balanced Scorecard (BSC) demonstra um sistema de medição completo do Modelo de Gestão de Incubadoras (CERNE), envolvendo as quatro perspectivas distintas sob a medição do desempenho, ou seja, o cliente (mercado), processos de negócio interno (gestão), financeiro (capital) e aprendizagem e inovação (tecnologia). As quatro perspectivas abrangem os diferentes aspectos do desempenho do CERNE: (i) a perspectiva do cliente busca enxergar como uma organização deve apresentar-se diante seus clientes; (ii) a perspectiva de processos internos busca encontrar o lado positivo da realização dos processos de negócios internos da empresa; (iii) a perspectiva financeira examina a melhor forma de apresentar-se de ante dos seus stakeholders e (iv) a perspectiva de aprendizagem e crescimento examina maneiras da organização aprender a realizar inovação, para sustentabilidade futura.

As medidas do BSC sustentam-se nos objetivos estratégicos da organização. Por meio da elaboração de uma série limitada de indicadoreschave de desempenho (indicadores críticos) para cada perspectiva, o painel de desempenho monitora o foco na visão estratégica. Enquanto as medidas utilizadas pelos sistemas tradicionais de avaliação fazem uso apenas de informações passadas (KAPLAN; NORTON, 1993).

O BSC oferece uma estrutura para a ligação de ativos intangíveis, criando valor para os acionistas mediante quatro perspectivas interrelacionadas (KAPLAN; NORTON, 2004). As perspectivas do BSC devem ser traduzidas em métricas de desempenho correspondentes e medidas que reflitam metas e objetivos estratégicos. As medidas 
adicionadas ao Balanced Scorecard devem ser monitoradas e avaliadas ao longo do tempo e integradas diretamente no modelo CERNE. A Figura 2 ilustra as práticas-chave do Sistema de Acompanhamento, Orientação e Avaliação alinhadas às perspectivas do Balanced Scorecard.

Figura 2 - Práticas-chave do sistema de acompanhamento, orientação e avaliação

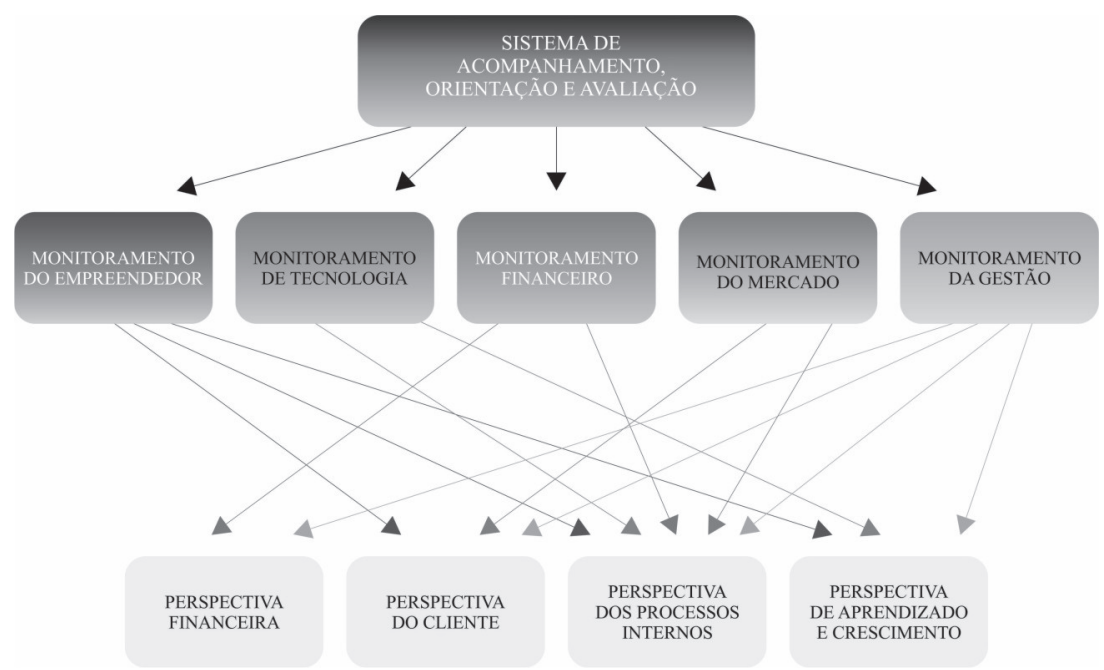

Fonte: Adaptado de Manual de Implantação CERNE (2011).

Analisando-se a Figura 2, percebe-se que o Balanced Scorecard apresenta-se como um instrumento capaz de auxiliar no monitoramento e avaliação da implantação das práticas-chave preconizadas pelo modelo CERNE. Evidenciar o capital intelectual tem sido objeto de estudo, nas últimas décadas, e continua desafiando pesquisadores. Estabelecer mecanismos de monitoramento e avaliação dos ativos intangíveis tem crescente importância à medida que se associada ao advento da economia do conhecimento. $\mathrm{O}$ estímulo às pesquisas que abranjam mecanismos de monitoramento ao capital intelectual decorre do impacto político do conhecimento no desempenho das organizações (RODRIGUES; DORREGO; JARDÓM-FERNÁNDEZ, 2009). 


\section{Metodologia}

Para desenvolvimento dessa pesquisa, adotou-se uma abordagem qualitativa mediante um estudo de caso de caráter exploratório em uma incubadora de base tecnológica. Segundo o Manual de Oslo (1997), recomenda-se que sejam coletados dados qualitativos sobre as atividades inovadoras, por se tratar de um curto período de observação. "Esta pesquisa tem por objetivo proporcionar maior familiaridade com o problema, com vista a torná-lo mais explícito ou a constituir hipótese" (GIL, 2007, p. 40), ou seja, esta pesquisa envolve levantamento bibliográfico, entrevistas com pessoas que têm experiências práticas com o problema pesquisado e análise de exemplos que estimulem a compreensão. "O estudo de caso é uma modalidade de pesquisa amplamente autorizada nas ciências biomédicas e sociais" (GIL, 2007, p. 54).

Para consecução da pesquisa e consequente construção dos objetivos estratégicos do mapa adotou-se como instrumento de coleta de dados primários um questionário de avaliação qualitativa o qual foi aplicado mediante entrevistas com os participantes da ITNC e com professores da área de negócios, a fim de obter informações sobre a opinião dos entrevistados, utilizando-se para tanto o método Delphi. Para construção dos objetivos estratégicos optou-se pela técnica Delphi, por possibilitar um processo participativo, em que os objetivos escolhidos para compor o Mapa e o Painel de Indicadores de desempenho repetiram-se a cada rodada, de modo que os participantes da incubadora pudessem reavaliar as suas respostas e as justificar.

Em linhas gerais, o método Delphi consulta um grupo de especialistas a respeito de eventos futuros através de um questionário, que é repassado continuadas vezes até que seja obtida uma convergência das respostas, um consenso, que representa uma consolidação do julgamento intuitivo do grupo. Pressupõe-se que o julgamento coletivo, ao ser bem organizado, é melhor do que a opinião de um só indivíduo. $O$ anonimato dos respondentes, a representação estatística da distribuição dos resultados, e o feedback de respostas do grupo para 
reavaliação nas rodadas subsequentes são as principais características deste método (WRIGHT; GIOVINAZZO, 2000, p.54).

Os dados secundários foram coletados nos documentos cedidos pela organização pesquisada e literatura especializada, os quais deram suporte aos dados primários. Na primeira rodada do Delphi os gestores das empresas incubadas foram questionados sobre quais os objetivos estratégicos críticos para desenvolvimento de seus respectivos negócios. Na segunda rodada, depois de compilados os resultados da primeira rodada, eles foram questionados dentre todos os objetivos estratégicos citados pelos demais dirigentes que compuseram o painel de especialistas, a assinalar com $X$ aqueles que se destacam como sendo os objetivos de maior importância dentro de cada uma das perspectivas do BSC.

A seguir, solicitou-se que os objetivos escolhidos fossem ordenados por grau de importância, sendo: 5 para o mais importante, 4 para importante, 3 para média importância, 2 para pouco importante e 1 para o menos importante. Na terceira rodada, já de posse dos objetivos estratégicos selecionados na rodada anterior, os empresários foram questionados se a partir daqueles objetivos seria possível traduzir a estratégia de sua empresa. Na quarta rodada a partir do mapa concebido contemplando os objetivos escolhidos, procurou-se alinhar às etapas do CERNE.

\section{Resultados da pesquisa}

\subsection{Incubadora Tecnológica Natal Central (ITCN)}

Pioneira no estado do Rio Grande de Norte, a incubadora tecnológica tem a finalidade de oferecer suporte e apoio ao surgimento de novas empresas de base tecnológica, principalmente nas áreas atuantes do Instituto ao qual a mesma está ligada. Conta com uma infraestrutura exclusiva de $700 \mathrm{~m}^{2}$, disponibilizando um espaço empreendedor para treze empresas em que cada empreendimento possui um espaço físico 
exclusivo de $24 \mathrm{~m}^{2}$, além de área coletiva com três salas de reuniões, sala de treinamento, copa, banheiro e sala de coordenação. As empresas também dispõem da infraestrutura institucional: laboratórios, bibliotecas, auditórios, restaurante, vigilância e estacionamento.

A incubadora definiu sua estrutura de gestão tomando como base a seguinte missão, visão e valores:

- Missão: apoiar o desenvolvimento de ideias inovadoras, transformando-as em empreendimentos de sucesso e contribuir para o desenvolvimento socioeconômico do Estado.

- Visão: ser reconhecida, até 2016, como uma incubadora de referência em empreendedorismo, inovação e desenvolvimento de novos negócios da região Nordeste.

- Valores: direcionada para resultados; comprometida; socialmente correta.

Os serviços oferecidos pela incubadora são iniciados antes mesmo da incubação de empresas, incluindo a sensibilização para o empreendedorismo, a prospecção de novos empreendimentos e a qualificação dos empreendedores. Durante o período de incubação, disponibilizam-se para as empresas assessorias e consultoria especializadas nas áreas financeira, tecnológica, mercado e gestão, com acompanhamento, avaliação e monitoramento dos empreendimentos em todo o processo de graduação. Até abril de 2014, a incubadora já graduou 16 empresas. Seu público alvo está direcionado aos estudantes do Campus Natal-Central, pesquisadores, desenvolvedores de novas tecnologias e projetos empreendedores.

\subsection{Indicadores de desempenho da incubadora}

Sabe-se que o monitoramento e a avaliação das empresas incubadas permitirão estabelecer parâmetros que se possa vir nortear a revisão e a elaboração das metas, e auxiliar na confecção de futuros editais para entradas de novas empresas, e permitam que estes sejam elaborados e executados com menores probabilidades de erros e, portanto, atingindo a eficácia almejada pela incubadora. 
O Quadro 1 apresenta o conjunto de indicadores atualmente adotados pela Incubadora para o monitoramento e avaliação das empresas incubadas de acordo com os cinco eixos do modelo CERNE.

\section{Quadro 1 - Monitoramento e avaliação das empresas incubadas - 2014}

\begin{tabular}{|c|c|c|c|}
\hline EIXO EMPREENDEDOR & \multicolumn{2}{|l|}{ EIXO TECNOLOGIA } & EIXO CAPITAL \\
\hline $\begin{array}{l}\text { Características do } \\
\text { Empreendedor } \\
\text { Persistência: } \\
\text { Costuma agir diante de } \\
\text { obstáculos significativos? } \\
\text { Quanto à estratégia. } \\
\text { Quanto aos objetivos e metas. } \\
\text { Comprometimento: } \\
\text { Grau de comprometimento } \\
\text { do empreendedor para com a } \\
\text { empresa } \\
\text { Tempo dedicado à empresa. } \\
\text { Comprometimento com cliente. } \\
\text { Riscos calculados: } \\
\text { Avalia alternativas e calcula } \\
\text { riscos deliberadamente? } \\
\text { Busca reduzir os riscos e } \\
\text { controlar seus resultados? } \\
\text { Encontra-se em situações } \\
\text { desafiantes ou de riscos } \\
\text { moderados? } \\
\text { Valores do empreendedor } \\
\text { atribuem valores pessoais à } \\
\text { empresa e à ITNC? } \\
\text { Ética. } \\
\text { Responsabilidade. } \\
\text { Pontualidade. }\end{array}$ & \multicolumn{2}{|c|}{$\begin{array}{l}\text { O processo produtivo vem se } \\
\text { desenvolvendo satisfatoriamente? } \\
\text { Evolução do processo. } \\
\text { Aumento da produtividade. } \\
\text { Os produtos vêm evoluindo } \\
\text { satisfatoriamente? } \\
\text { Evolução dos produtos. } \\
\text { Melhorias da qualidade. } \\
\text { Inovação do produto em relação } \\
\text { aos concorrentes? } \\
\text { Inovação. } \\
\text { Tem investido em PD\&l? } \\
\text { Número de ideias executadas. }\end{array}$} & $\begin{array}{l}\text { A empresa tem obtido } \\
\text { retorno sobre o capital } \\
\text { ou recursos investidos no } \\
\text { negócio? } \\
\text { Retorno sobre o } \\
\text { investimento. } \\
\text { Retorno sobre vendas. } \\
\text { A empresa tem alcançado } \\
\text { crescimento dos lucros no } \\
\text { último período? } \\
\text { Lucratividade. } \\
\text { Taxa de crescimento. } \\
\text { Geração e capitação de } \\
\text { recursos. } \\
\text { Investimentos. } \\
\text { Capital de giro. } \\
\text { Composição do capital } \\
\text { \% Recursos próprios. } \\
\text { \% Recursos de terceiros. }\end{array}$ \\
\hline \multicolumn{2}{|l|}{ EIXO MERCADO } & \multicolumn{2}{|c|}{ EIXO GESTÃO } \\
\hline \multicolumn{2}{|c|}{$\begin{array}{l}\text { A empresa possui um plano de mercado? } \\
\text { Execução do plano de marketing: promoção (como) e } \\
\text { vendas (quanto). } \\
\text { Investimento em marketing. } \\
\text { Como a empresa realiza a segmentação de mercado? } \\
\text { Identificação de clientes alvos. } \\
\text { Reconhecimento de mercados em potencial e } \\
\text { capacidade para atrair novos clientes. } \\
\text { Quais os recursos que a empresa utiliza para divulgar e } \\
\text { consolidar seu produto? } \\
\text { Criação de marca e material gráfico. } \\
\text { Desenvolvimento de portfólio, folders e banners. } \\
\text { A empresa dispõe de sistemática para } \\
\text { acompanhamento da satisfação do cliente? } \\
\text { Utilização das informações do SAC para implementação } \\
\text { de melhorias nos produtos ou processos. } \\
\text { Pesquisa e identificação de necessidades. }\end{array}$} & \multicolumn{2}{|c|}{$\begin{array}{l}\text { Produto/Negócio } \\
\text { Estágio de desenvolvimento do produto } \\
\text { Lançamento de novos produtos ou serviços } \\
\text { Planejamento e gestão } \\
\text { Definição dos objetivos estratégicos. } \\
\text { Plano de ação com responsáveis e prazos. } \\
\text { Mercado } \\
\text { Número de clientes ativos? } \\
\text { Conhecimento dos concorrentes diretos e } \\
\text { indiretos. } \\
\text { Pessoas } \\
\text { Número de colaboradores? } \\
\text { Plano de descrição de cargos/funções }\end{array}$} \\
\hline
\end{tabular}

Fonte: Dados da Pesquisa (2014). 
Segundo o Manual de Implantação do CERNE (2011), o sistema de acompanhamento, orientação e avaliação das empresas, envolve a sustentação de um processo sistemático documentado e avaliado, que permite a incubadora monitorar e orientar o desempenho de seus empreendimentos nos cinco eixos de progressão do negócio: empreendedores, gestão, produto, mercado e recursos.

Para implantação desse processo-chave, a incubadora deve apresentar a realização periódica e sistematizada do acompanhamento, avaliação e orientação das empresas incubadas (CERNE, 2011).

Para fins de ilustração, o Quadro 2 apresenta o painel de indicadores do ciclo completo de um dos cinco eixos de monitoramento do processo-chave 1.6 e na sessão seguinte evidencia-se como se daria sua integração às premissas do Balanced Scorecard. 
Quadro 2 - Eixo Tecnologia

\begin{tabular}{|c|c|c|}
\hline \multicolumn{3}{|l|}{2 - EIXO TECNOLOGIA } \\
\hline \multicolumn{3}{|c|}{ 2.1 Resultados apresentados pelos indicadores tecnológicos da empresa: } \\
\hline \multicolumn{3}{|c|}{ a) O processo produtivo vem se desenvolvendo satisfatoriamente? } \\
\hline i. Evolução do processo & Pontuação (1 a 5) & \\
\hline ii. Aumento de produção & Pontuação (1 a 5) & \\
\hline iii. Aumento da produtividade & Pontuação (1 a 5) & \\
\hline \multicolumn{3}{|l|}{ Observações: } \\
\hline \multicolumn{3}{|l|}{ b) Os produtos vêm evoluindo satisfatoriamente? } \\
\hline iv. Evolução dos produtos & Pontuação (1 a 5) & \\
\hline v. Redução de custos & Pontuação (1 a 5) & \\
\hline vi. Melhorias da qualidade & Pontuação (1 a 5) & \\
\hline \multicolumn{3}{|l|}{ Observações: } \\
\hline \multicolumn{3}{|l|}{ c) Inovação do produto em relação aos concorrentes? } \\
\hline vii. Tem inovação & Pontuação (1 a 5) & \\
\hline viii. Não tem inovação & Pontuação (1 a 5) & \\
\hline \multicolumn{3}{|l|}{ Observações: } \\
\hline d) Tem investido em PD\&I? & \multicolumn{2}{|l|}{ Observações: } \\
\hline ix. Número de ideias que chegaram a ser executadas & Pontuação (1 a 5) & \\
\hline x. Número de ideias executadas que tiveram sucesso & Pontuação (1 a 5) & \\
\hline \multicolumn{3}{|l|}{ Observações: } \\
\hline \multicolumn{3}{|c|}{ e) A equipe da empresa tem qualificação adequada às suas necessidades? } \\
\hline xi. Graduação & Pontuação (1 a 5) & \\
\hline xii. Especialização & Pontuação (1 a 5) & \\
\hline xiii. Mestrado & Pontuação (1 a 5) & \\
\hline xiv. Doutorado & Pontuação (1 a 5) & \\
\hline \multicolumn{3}{|l|}{ Observações: } \\
\hline \multicolumn{3}{|c|}{ f) A empresa possui os softwares necessários para execução do produto? } \\
\hline xv. Grau de necessidade dos softwares & Pontuação (1 a 5) & \\
\hline xvi. Quantidade de softwares & Pontuação (1 a 5) & \\
\hline Observações: & & \\
\hline
\end{tabular}

Fonte: Dados da pesquisa (2014).

Os resultados apresentados pelos indicadores de cada eixo são avaliados a partir de um conjunto de perguntas, cada uma contendo um conjunto de itens que recebem pontuação individual variável entre 1 e 5 pontos. 


\subsection{Mapa estratégico e BSC da incubadora}

Os objetivos estratégicos foram definidos utilizando-se a técnica Delphi, conforme explicitado na metodologia. Procurou-se então alinhar os indicadores de desempenho dos processos-chave de monitoramento do modelo CERNE aos objetivos estratégicos identificados de forma a instituir o mapa estratégico e o painel de indicadores (Balanced Scorecard) adequados às necessidades da incubadora. $O$ resultado encontra-se apresentado na Figura 3.

Figura 3 - Mapa estratégico e BSC da Incubadora Tecnológica Natal

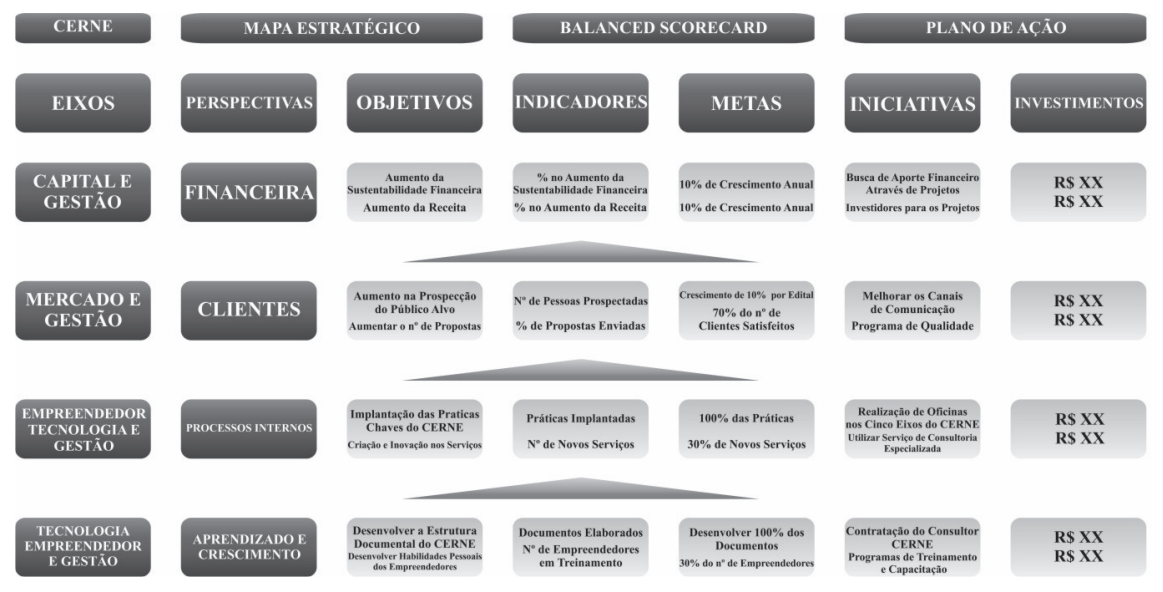

Fonte: Autores (2014).

A primeira coluna do mapa estratégico corresponde aos eixos do CERNE; e a segunda coluna, às correspondentes perspectivas do BSC.

\section{Considerações finais}

A pesquisa buscou propor um modelo de gestão aplicável às incubadoras, tendo por base os mapas estratégicos e painéis de desempenho à luz das premissas do Balanced Scoredcard (BSC) e utilizá-lo como ferramenta de medição de desempenho das empresas incubadas de acordo com as práticas-chaves do modelo CERNE. 
Realizou-se um estudo de caso em uma incubadora de base tecnológica. O uso da abordagem qualitativa e da técnica Delphi mostrou-se adequado ao escopo do trabalho, tendo em vista que permitiu a construção do conjunto de objetivos estratégicos os quais foram utilizados para composição do Mapa e do Painel de Indicadores de Desempenho demonstrados na sessão anterior. Esta pesquisa contribui com evidências empíricas sobre a implantação de mecanismos de mensuração de desempenho adaptáveis ao contexto das incubadoras tecnológicas.

As limitações da pesquisa são inerentes à metodologia adotada, o estudo de caso, e, portanto a mesma não é passível de generalizações. Entretanto, os resultados da pesquisa revelam uma proposta inicial de mapa e painel de desempenho, contemplando as quatro perspectivas propostas pelo BSC (aprendizagem e desenvolvimento, processos internos, clientes e financeira).

Por se tratar de uma abordagem inovadora da gestão do relacionamento e de avaliação de desempenho entre a incubadora e as empresas beneficiadas pelo sistema, recomenda-se para estudos futuros a elaboração de um mapa estratégico, contemplando todos os eixos do modelo CERNE, e que sejam realizados estudos comparativos a outros modelos de implantação não contemplados nesta pesquisa.

\section{Referências}

BRASIL. Associação Nacional de Entidades Promotoras de Empreendimentos Inovadores -ANPROTEC (org.). Disponível em: <>http://www.anprotec.org.br>. Acesso em: 15 jan. 2011.

. Centro de Referência para Apoio a Novos Empreendimentos. Associação Nacional de Entidades Promotoras de Empreendimentos Inovadores. Brasília, DF: ANPROTEC, 2011.

CONH, J.; KHURANA, R. Mapas estratégicos para planejamento da sucessão do CEO. In. Balanced Scorecard: reflexões, idéias \& 
experiências para organização orientadas para estratégias, Boston: HarvardBusiness School Publishing, 2003.

COSTA, A. P. P. Balanced Scorecard: conceitos e guias de implementação. São Paulo: Atlas, 2006.

FERNANDES, B. H. R.; BERTON, L. H. Administração Estratégica: da competência empreendedora à avaliação de desempenho. São Paulo: Saraiva, 2005.

GIL, A. C.; Como elaborar um projeto de pesquisa. 4. ed., São Paulo: Atlas, 2007.

IGARASHI, D. C. C.; IGARASHI, W.; FERREIRA, E. S.; JÚNIOR, J. B. H.. Implantação do mapa estratégico e painel de desempenho em uma empresa comercial da região oeste do Paraná. RIC - Revista de Informação Contábil, Recife, v. 4, n. 4, p. 23-41, out-dez. 2010.

KAPLAN, R. S.; NORTON, D. Putting the balanced scorecard to work. Harvard Business Review, Boston, v.71, n.5, , p. 134-147, sep./oct. 1993.

Janeiro: Elsevier, 1997.

. A estratégia em ação: balanced scorecard. 24. ed. Rio de . Measuring the strategic readiness of intangible assets. Harvard Business Review, Boston, v.82, n.2, p. 52-63, Feb. 2004.

- Alinhamento: utilizando o balanced scorecard para criar sinergias corporativas. Rio de Janeiro: Elsevier, 2006.

Organização orientada para a estratégia: como as empresas que adotam o balanced scorecard prosperam no novo ambiente de negócios. Rio de Janeiro: Campus, 2000.

MANUAL DE OSLO. Diretrizes para a coleta e interpretação de dados sobre Inovação. Tradução FINEP. 3. ed. Rio de Janeiro: FINEP, 2007. p. 184. Disponível em: <http://www.uesc.br/nucleos/nit/manualoslo. pdf>. Acesso em :15 jan. 2011. 
REZENDE, J.F. Balanced Scorecard e a gestão do capital intelectual: alcançando a performance balanceada na economia do conhecimento. 2. ed. Rio de Janeiro: Elsevier, 2003.

RODRIGUES, H. M. S. S.; DORREGO, P. F. F.; JARDÓM-FERNÁNDEZ, C. M. F.. Enla Capacidad de Innovación de las Empresas del Sector de Automoción de la Eurorregión Galicia Norte de Portugal, Vigo: Universidade de VIGO, 2009.

SERVIÇO BRASILEIRO DE APOIO ÀS MICRO E PEQUENAS EMPRESAS (SEBRAE). Benefícios para os pequenos negócios. Minas Gerais: Sebrae, 2011.

WRIGHT, J. T. C.; GIOVINAZZO, R. A.. DELPHI: uma ferramenta de apoio ao planejamento prospectivo.Revista de Gestão: Caderno de Pesquisas em Administração, São Paulo, v. 1, n. 12, p. 54-65, $2^{\circ}$ Trimestre. 2000. Disponível em: <http://www.fundacaofia.com.br/ profuturo/Uploads/Documents/Artigos/art50.htm>. Acesso em: 26 maio 2014.

Artigo recebido em: 05/03/2015

Aprovado em: 12/06/2015 